\title{
Precise lattice parameters through distortions refinement using circular harmonics
}

\author{
P. Brázda, L. Palatinus \\ Institute of Physics of the Czech Academy of Sciences, Na Slovance 1999/2, 18200 Prague 8, \\ brazda@fzu.cz
}

3D electron diffraction (3D ED) undergoes rapid development in the past years. Structure solution is relatively easy, dynamical refinement provides accurate structure models and also absolute structure determination [1], but the accuracy of the lattice parameters remains a problem despite some effort. Lattice parameters obtained by 3D ED have at least an order of magnitude lower accuracy than single crystal $\mathrm{x}$-ray data and the comparison is even worse with powder x-ray data. The reasons causing this poor accuracy are instrument-induced geometrical distortions present in the data and, in case of beam sensitive samples, crystal damage induced by the electron beam. In 2D diffraction patterns, distortions caused by aberrations of electromagnetic lenses are well known and have been analysed several times [2], but the precession assisted data collection induces new distortion, which were never analysed and we quantify and describe them for the first time._For mathematical description, we split the total in-plane difference between the expected and observed position of the diffraction maxima into radial and tangential ( $\Delta r$ and $\Delta t$ ) contributions, which are then described by circular harmonics - function of diffraction vector length $r$ and azimuth $\varphi$ of the diffraction maximum (eq. 1).

$$
\Delta r=\sum_{n=0}^{N}\left(\cos \left(n\left(\varphi-\varphi_{r, n}\right)\right) \sum_{m=1}^{M} \rho_{n, m} r^{m}\right) \quad \Delta t=\sum_{n=0}^{N}\left(\cos \left(n\left(\varphi-\varphi_{t, n}\right)\right) \sum_{m=1}^{M} \tau_{n, m} r^{m}\right)
$$

Parameters $\varphi_{r, n}, \varphi_{t, n}, \rho_{n, m}$ and $\tau_{n, m}$ need to be determined either by the calibration of the microscope or by the refinement against the diffraction data. This general approach allowed us to compensate for all observed distortions, not only the classical pincushion-barrel, spiral and elliptical. The distortions depend on excitation of the lenses and can be calibrated. Tools for distortion refinement are incorporated in software PETS 2 [3] and distortion refinement workflow may be found in Jana Cookbook example Borane [4].

The effects of geometrical distortions in 3D ED data was so far only marginally analysed [3]. Here we analyse the effects of both the distortions in the plane of diffraction image and also the errors in the crystal orientation. The effect of the accumulated electron dose is also significant (Figure 1). Measurements of anti- $\mathrm{B}_{18} \mathrm{H}_{22}$ molecular crystal shows that the beam damage results in lattice parameters increase by about $1.5(5) \%$ per accumulated dose of $1 \mathrm{e}^{-} . \AA^{-2}$.
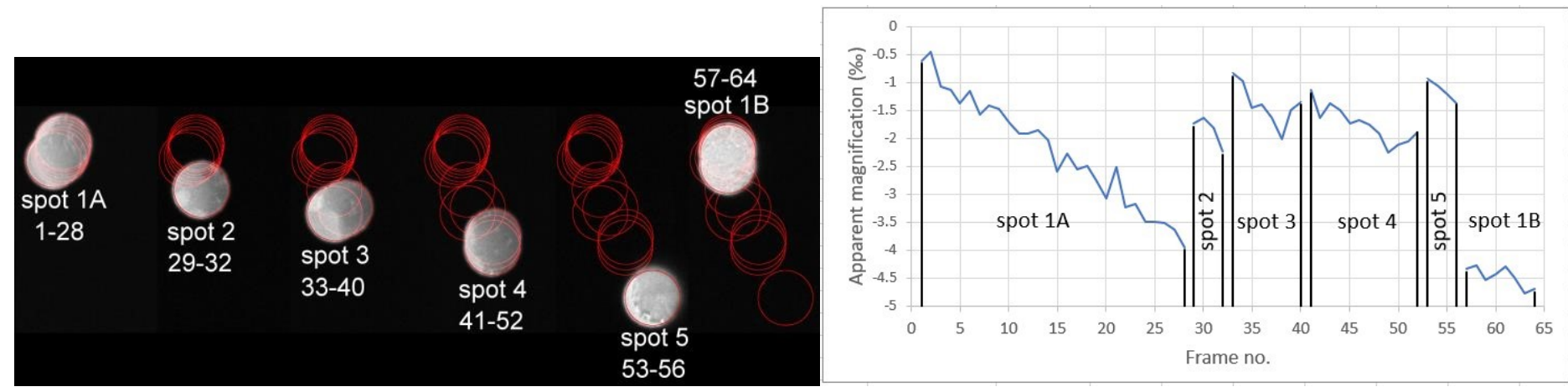

Figure 1. Left: beam scan of crystal of anti- $\mathrm{B}_{18} \mathrm{H}_{22}$ with marked frame numbers. Right: apparent magnification changes (reciprocal space) due to crystal deterioration.

1. Palatinus, L., et al., (2017) Science 355, 166; Brázda, P., Palatinus, L., Babor, M., (2019) Science 364, 667.

2. Capitani, G. C., Oleynikov, P., Hovmöller, S., Mellini, M., (2006) Ultramicroscopy 106, 66.

3. Palatinus, L., et al., Acta Crystallogr. (2019) B75, 512.

4. http://jana.fzu.cz/.

Keywords: 3D ED, accurate lattice parameters, distortions, beam damage, precession, frame orientation

This research was supported by the Czech Science Foundation, project number 21-05926X. We acknowledge CzechNanoLab Research Infrastructure supported by MEYS CR (LM2018110). 